\title{
Identificación de costos ocultos a partir de un estudio de organización del trabajo en una empresa del sector farmacéutico en Cuba
}

\author{
Identification of hidden costs from the work organization study of an enterprise \\ from the pharmaceutical sector in $\mathrm{Cuba}$
}

\author{
Ana María Negrón González ${ }^{1}$ 2* $^{*}$ María Sonia Fleitas Triana ${ }^{1} \quad$ Germán Gémar Castillo $^{3}$ \\ José Carlos Negrón González ${ }^{1} \quad$ Vania García Fenton ${ }^{1} \quad$ Yoimi Trujillo Reyna $^{1}$
}

Recibido 18 de diciembre de 2015, Aceptado 20 de marzo de 2017

Received: June 18, 2015 Accepted: March 20, 2017

\begin{abstract}
RESUMEN
La organización del trabajo juega un papel fundamental en la eficiencia de los procesos, diversos autores plantean la necesidad de evaluar su impacto en los resultados de la empresa a través de indicadores de gestión. Por otra parte, la Organización Internacional de Trabajo ha definido los tiempos que pueden generar en derroches para la producción. Además, estudios franceses han mostrado que la incorrecta gestión del tiempo puede generar disfuncionamientos y estos incidir en costos ocultos. Todos estos elementos muestran como el análisis siempre es desde una visión estratégica y no con una fundamentación técnica desde la organización del trabajo. Es por ello que los autores no explican o muestran procedimientos o metodologías de cómo identificar los disfuncionamientos y examinarlos, por lo cual esta investigación analiza como cuantificar los costos ocultos asociados a las disfuncionalidades existentes en el proceso de organización del trabajo para evitar que estos afecten a la productividad y como objetivo: identificar los costos ocultos a partir de un estudio de organización del trabajo en una empresa del sector farmacéutico en Cuba. Las técnicas fundamentales utilizadas son la entrevista, la encuesta y la lista de chequeo. En la indagación se detectó como principales disfuncionamientos: la inexistencia de instrumentos y herramientas necesarias y los tiempos improductivos por indisciplina laboral, este último genera altos costos, para ello se proponen indicadores que permiten monitorear su comportamiento. El procedimiento aplicado aporta una herramienta para controlar los disfuncionamientos existentes en la organización del trabajo y sus costos a través de indicadores.
\end{abstract}

Palabras clave: Organización del trabajo, procedimiento, disfuncionamientos, costos ocultos.

\section{ABSTRACT}

Work Organization plays a fundamental role in process efficiency; several authors suggest the need to evaluate its impact on company results through management indicators. In addition, french studies have shown that time administration can cause malfunctions and influence in these hidden costs. Moreover, the International Labor Organization has defined the time types generating wastes in production. All these elements show how the analysis is always from a strategic vision and not with a technical basis for the organization of work. These authors do not explain, or show procedures or methodologies on how to

\footnotetext{
1 Ingeniería Industrial. Universidad Tecnológica de La Habana “José Antonio Echevarría”. CUJAE. Calle 114 N $11901 \%$ Ciclo vía y Rotonda, Marianao, La Habana, Cuba. E-mail: anamaria@ind.cujae.edu.cu; sfleitas@ind.cujae.edu.cu; negron@dcrhmail.cujae.edu.cu; vania@ind.cujae.edu.cu,vreu@tesla.cujae.edu.cu

2 Programa de Doctorado en Economía y Administración de Empresas. Universidad de Málaga. Avda. Cervantes, 229.071. Málaga, España. E-mail: anamarianegrong@uma.es

3 Economía y Administración de Empresas. Universidad de Málaga. Málaga, España. E-mail: ggemar@uma.es

* Autor de correspondencia
} 
identify and examine the dysfunctions. Thus, this investigation is aiming at analyzing and quantifying the dysfunctions of the work organization process and to prevent that these dysfunctions affect productivity. The Goal of the investigation is to identify the hidden costs through a work organization study in an enterprise of the pharmaceutical sector in Cuba. The tools employed are the interview, the surveys and checklists. IT was detected through the exploration the main dysfunctions are associated to the nonexistent instruments and necessary tools and the unproductive times caused by lack of work discipline, which generates high level of cost. Consequently, the investigation proposes indicators to monitor the labor process. The procedure applied constitutes a tool to control the existing dysfunctions and costs of the work organization process through indicators.

Keywords: Work organization, procedure, dysfunctions and hidden cost.

\section{INTRODUCCIÓN}

Los principios de la administración científica es la obra fundamental de Taylor en la cual se aborda la organización del trabajo y de la producción, y se instauran reglas, sistemas contables y de medición con el propósito de elevar la productividad del trabajo y los volúmenes de producción [1]. Es por todo esto que Taylor es considerado uno de los principales iniciadores de la organización del trabajo en su manifestación práctica.

La organización del trabajo permite relacionar de la mejor forma posible la técnica y las personas en el proceso de producción, que garantice el uso más efectivo de los recursos materiales y laborales [2]. También permite aprovechar al máximo los recursos y obtener la mayor producción posible con el mínimo de insumos [3].

Autores como Niebel [4]; Hodson [5] y Marsán [6] se distinguen por el estudio de la gestión de las personas en estrecha relación con la organización del trabajo, con marcado énfasis en sus dos pilares fundamentales: el estudio de los tiempos y los métodos de trabajo.

En Cuba a la gestión de recursos humanos en las empresas se le han incorporado nuevos enfoques dirigidos a gestionar de forma integrada todos los procesos asociados al capital humano para alcanzar un desempeño superior y aumentar la productividad del trabajo, para ello se implementa en las organizaciones el modelo de Morales [7] también desarrollado en las Normas Cubanas 3000: 3002 [8-10], en sus 9 módulos, donde un proceso esencial es la organización del trabajo y las competencias son el centro de la gestión para las organizaciones [11-12].
En la gestión de los recursos humanos existen disfuncionamientos conceptualizados como la "diferencia existente entre el funcionamiento esperado de la misma y el funcionamiento real, como resultado de las interacciones entre las estructuras de la organización y los comportamientos humanos" [1].

Esta investigación se centra en: identificar los disfuncionamientos que se generan dentro de la organización del trabajo en los procesos y cómo estos pueden contribuir a la existencia de costos que no son cuantificados en los estados financieros.

Es importante destacar que una correcta gestión para la diminución de los disfuncionamientos permite alcanzar niveles superiores en el desempeño laboral, así como un incremento de la productividad del trabajo, potenciando las variables sociales de estas organizaciones, y aprovechándolas al máximo para aumentar los ingresos. Las técnicas utilizadas son: la entrevista, el análisis documental, la encuesta, la lista de chequeo, entre otras.

\section{ANÁLISIS TEÓRICO}

\section{Antecedentes de la organización del trabajo}

La dirección de recursos humanos en las empresas se caracteriza como una función eminentemente directa, macro organizacional, dinámica y en constante transformación [13].

El factor humano se encuentra en todos los momentos del hecho productivo, puesto que se requiere de la participación de las personas y de una constante relación social laboral entre ellas, [14]. Aunque también se hace necesario para mantener la supervivencia de las empresas, que las mismas asuman de lleno la dinámica del aumento 
en la frecuencia de introducción de productos, la reducción de tiempo y la optimización en los procesos de desarrollo [15].

Es por ello que la gestión del factor humano está dada en gran parte por la gestión integrada del capital humano y un proceso esencial en esta gestión es la organización del trabajo que puede definirse como el conjunto de aspectos técnicos y sociales que intervienen en la producción de bienes y servicios [16].

También se ha considerado que la organización del trabajo es una variable independiente que tributa directamente al grado de satisfacción con el trabajo, [17] y al aumento de la productividad del trabajo. Esta última se refiere a la relación establecida entre la cantidad de bienes o servicios producidos y la cantidad de recursos utilizados para producirlos ellos [18].

La organización del trabajo con sus herramientas y métodos es utilizado en diferentes sectores de la sociedad como por ejemplo en la medicina para establecer un "plan de operaciones". Este plan debe contemplar al menos la secuencia de las actividades, los recursos necesarios, la distribución de responsabilidades entre los profesionales y el calendario o cronograma de las actividades [19].

La literatura especializada muestra tres modelos fundamentales que explican cómo la organización del trabajo es realizada por el capital contemporáneo [20]:

1. La clásica organización de Taylor - Ford, generalmente asociada con la producción en masa.

2. La producción "Lean", inspirada en el modelo japonés también conocido como Just in Time.

3. Organizaciones flexibles y autónomas, inspiradas en la Escuela Sociotécnica.

La organización de Taylor - Ford busca la racionalización del trabajo. Por lo tanto, las principales características definen este tipo de organización son: la división del trabajo, tanto en sentido horizontal (subdivisión de tareas) y verticalmente (separación entre concepción y ejecución); la especialización de las tareas; la supervisión constante; subdividen las tareas de trabajo individuales, por lo que es adecuada para la producción a gran escala [21].
Organizaciones eficientes que se centran en crear más valor para los clientes con menos recursos se caracterizan por el enriquecimiento del trabajo y el trabajo en equipo. Este consiste en ampliar el contenido del trabajo por métodos tales como: la rotación de los puestos de trabajo, así como la expansión horizontal y vertical del mismo. Tales organizaciones a menudo han enriquecido grupos por inspirado el modelo de Toyota con las siguientes características: la organización del grupo con relativa autonomía, la responsabilidad y la versatilidad de los trabajadores, el énfasis en las mejoras operativas confinados al lugar de trabajo y la adopción sistemática de las técnicas de gestión derivados de Control Total de Calidad (CTC) [22].

Las organizaciones flexibles y autónomas se basan en la escuela sociotécnico, que aboga por la formación de los llamados grupos semiautónomos. Este tipo de organización reúne a los objetivos y las responsabilidades comunes comparten con los empleados Los trabajadores son lo tanto responsable de la definición de la forma de trabajo se va a realizar; de establecer los objetivos que se han de cumplir y; de determinar los recursos necesarios para llevar a cabo el trabajo para alcanzar las metas establecidas. Por lo tanto, se cree que es el modelo de organización proporciona más autonomía a los trabajadores [23].

Diferentes autores han desarrollado procedimientos para el estudio de la organización del trabajo en los procesos de producción, los cuales permiten organizar los elementos esenciales para optimizar los procesos, entre ellos se destaca: la Organización Internacional del Trabajo (OIT), la cual presenta un procedimiento con ocho etapas fundamentales, las cuáles comienza con seleccionar el proceso que se ha de estudiar, luego registrar los datos relevantes del mismo y posteriormente examinar los hechos registrados con espíritu crítico, así como evaluar los resultados obtenidos con el nuevo método en comparación con la cantidad de trabajo necesario y establecer un tiempo tipo [3].

Mientras Niebel [4] propone siete pasos o etapas esenciales acogiéndose al procedimiento de la OIT, donde propone seleccionar el proyecto, obtener y analizar los datos, desarrollar el método ideal, establecer el mismo, así como desarrollar un análisis del trabajo y establecer tiempos estándar. 
Los procedimientos desarrollados en Cuba tienen en su base el método general de solución de problema, desarrollado por Marsán en 1987 [6], los cuáles desarrollan la aplicación de este método adaptado al estudio del trabajo los diferentes pasos: definición del problema, análisis del problema, búsqueda de soluciones, evaluación de alternativas de solución, elección de la mejor solución, implementación de la solución y por último evaluar efectividad y rediseñar los procesos.

Un ejemplo de ello, es Basnuevo [24] que desarrolla un procedimiento basado en la mejora continua para la búsqueda de la eficiencia productiva, este procedimiento tiene 4 etapas y define en cada una de ellas las técnicas a aplicar para el cumplimiento de las mismas.

Bernal y Ramos [25] desarrollan un procedimiento partiendo de tres pasos fundamentales que son comunes para los procedimientos mencionados anteriormente: análisis del proceso, diagnóstico de los problemas, búsqueda y evaluación de las soluciones.

Otro procedimiento que adopta los pasos del método general de solución de problemas es el propuesto por Fleitas y García [26], y a través de estos desarrollan todos requisitos del módulo de organización del trabajo de la Norma Cubana (NC) 3001, 2007 [9].

En este procedimiento cuando se analiza problema se estudia el proceso para detectar cual es el valor agregado e identificar los derroches existentes, así como para cada una de las actividades identificadas y se determina cuáles no agregan valor al producto o servicio y aquellas en las que se gasta más tiempo o recursos que los necesarios [26].

En este procedimiento están implícitas las técnicas para la realización del estudio del proceso, los métodos y la medición del trabajo sugerido en la NC 3002/2007 [10] y en la etapa de búsqueda de soluciones se concreta la innovación organizacional resultante del estudio de organización del trabajo. Otro elemento que lo destaca es la planificación del estudio que se realiza utilizándose Microsoft Project, una herramienta muy eficaz para la planificación de los estudios [26].

En los procedimientos mencionados anteriormente no se muestran cómo se realizan la evaluación económica de la organización del trabajo, aunque Kanawaty [3] menciona la importancia de la búsqueda de métodos de trabajo más económicos. Otros procedimientos como Marsán, Cuesta, García y Padilla [6] y Basnuevo, [24] plantean en el análisis del problema que se debe tener en cuenta las consideraciones de índole económica y Fleitas y García [26] en este paso plantea la necesidad de la identificación de los derroches, así como determinar las actividades que no agregan valor al producto o servicio y aquellas en las que se gasta más tiempo o recursos que los necesarios.

\section{METODOLOGÍA}

A partir de los análisis realizados a los diferentes modelos y procedimientos estudiados se rediseña el procedimiento de Fleitas y García [26], para lo cual se tiene en cuenta los pasos a seguir para detectar los derroches y para ello se utiliza la identificación de los disfuncionamientos que generan los mismos como se muestra en la Figura 1.

Los disfuncionamientos según Savall y Zardet [27] se agrupa en 6 familias, una de ellas es la gestión del tiempo uno de los pilares fundamentales de la organización del trabajo, por lo que en investigaciones anteriores ya se había planteado como se pueden detectar los disfuncionamientos en cada uno de los procesos de la gestión de los recursos humanos y por ende los asociados a la organización del trabajo [28].

Para esta investigación se aplica en una empresa del sector farmacéutico el procedimiento presentado por Fleitas y García, [26] modificado pues se le incorpora la identificación de los disfuncionamientos y la cuantificación de los costos ocultos. Esto revela mayor importancia cuando a diferencia de los modelos anteriores estudiados se parte de los elementos técnicos que se agrupan a través de los principios de la organización científica del trabajo, además de las formas de calcular los costos ocultos y los indicadores para controlar los mismos.

El procedimiento de Fleitas y García [26], consta de 7 etapas con actividades en cada una de ella. Las mismas se relacionan a continuación:

\section{Definición del problema:}

Para dar cumplimiento al requisito 4.3.1 se identifican los procesos que añaden valor o encarecen los costos, 
a partir del análisis de los indicadores económicos y de calidad. En la determinación de las premisas se identifican el usuario o cliente, según corresponda, se determinan los atributos del producto, servicio, el valor agregado, que exige el usuario o cliente, así como los procesos que respaldan estos atributos. También se definen los sistemas de organización que respaldan los procesos.

Para el cumplimiento del requisito 4.3.2 se exige que se elabore y apruebe el programa para el estudio del trabajo y la planificación de las siete etapas del método general de solución de problemas para garantizar el análisis del proceso. Así como a partir del requisito 4.3 .3 se designa y aprueban los responsables y participantes en el estudio del trabajo con una representación de los trabajadores. Posteriormente se conforma el equipo y se informa a los trabajadores sobre el estudio a realizar y los participantes en el mismo.

\section{Análisis del problema}

Requisito 4.3.4 y Requisito 4.3.5. Las técnicas empleadas en esta etapa tienen adecuaciones para los distintos tipos de procesos de trabajo. Una vez definido el problema se establecen los pasos para el estudio de los procesos, los métodos y la medición del trabajo.

El estudio de procesos se realiza a través del análisis del diagrama del proceso de operación, transporte, inspección, demora y almacenamiento (OTIDA) y se realiza el análisis del valor agregado e identificación de los derroches.

A partir de estudio de métodos se describen los métodos de trabajo utilizados en los puestos de trabajo, se analiza la organización y servicio al mismo, así como la determinación de las condiciones técnico-organizativas y de trabajo a través de un examen crítico de cada una de las operaciones del proceso.

En la medición del trabajo: se realizan las observaciones continuas individuales para la determinación del aprovechamiento de la jornada laboral (AJL). También se determinan y planifican las reservas de productividad, así como los indicadores de eficiencia.

A partir de los problemas encontrados en el estudio realizado se aplica la lista de chequeo que se muestra en la Tabla 1. En esta técnica se agrupan los disfuncionamientos a través de los 7 elementos de la organización científica del trabajo: división y cooperación del trabajo, método del trabajo, organización y servicio del puesto de trabajo, condiciones de trabajo, normación del trabajo, organización de los salarios y disciplina laboral [6].

A partir de los disfuncionamientos encontrados se cuantifican los costos por el método de costeo $\mathrm{ABC}$, desglosándolos por ítems de actividades que componen cada disfuncionamiento encontrado en el proceso.

Partiendo del análisis del problema se realiza un informe del diagnóstico y se exponen los resultados al colectivo de trabajadores para recibir retroalimentación sobre este.

\section{Búsqueda de soluciones}

Para dar cumplimiento al Requisito 4.3.6 se propone una descripción detallada del proceso de trabajo o método a aplicar y a partir de ello las herramientas y equipos que se utilizarán, así como de las condiciones de trabajo, de seguridad y salud en el trabajo y ergonómicas a garantizar. También se elabora el diagrama de la disposición del lugar de trabajo y posible croquis de las herramientas, plantillas y otros dispositivos.

En estos pasos se concreta el nuevo diseño del proceso y los métodos de trabajo a emplear en los diferentes puestos, lo que constituye la innovación organizacional resultante del estudio de organización del trabajo.

Para dar cumplimiento al requisito 4.3 .7 se determinan los tiempos de trabajo que debe invertir el trabajador competente en llevar a cabo una tarea según una norma de rendimiento, tiempo o servicio preestablecida y actualizada. También el aprovechamiento de la jornada laboral que se proyecta alcanzar y el número de trabajadores y los equipos requeridos para el cumplimiento de las tareas según el balance de carga y capacidad.

Para el procedimiento para el diseño del proceso de organización del trabajo se tiene en cuenta los elementos planteado por Rodríguez [24], con su tecnología de aprendizaje organizacional para la implementación del sistema de gestión integrada del capital humano y los elementos que contribuyen al 
Tabla 1. Lista de Chequeo para la identificación de los disfuncionamientos.

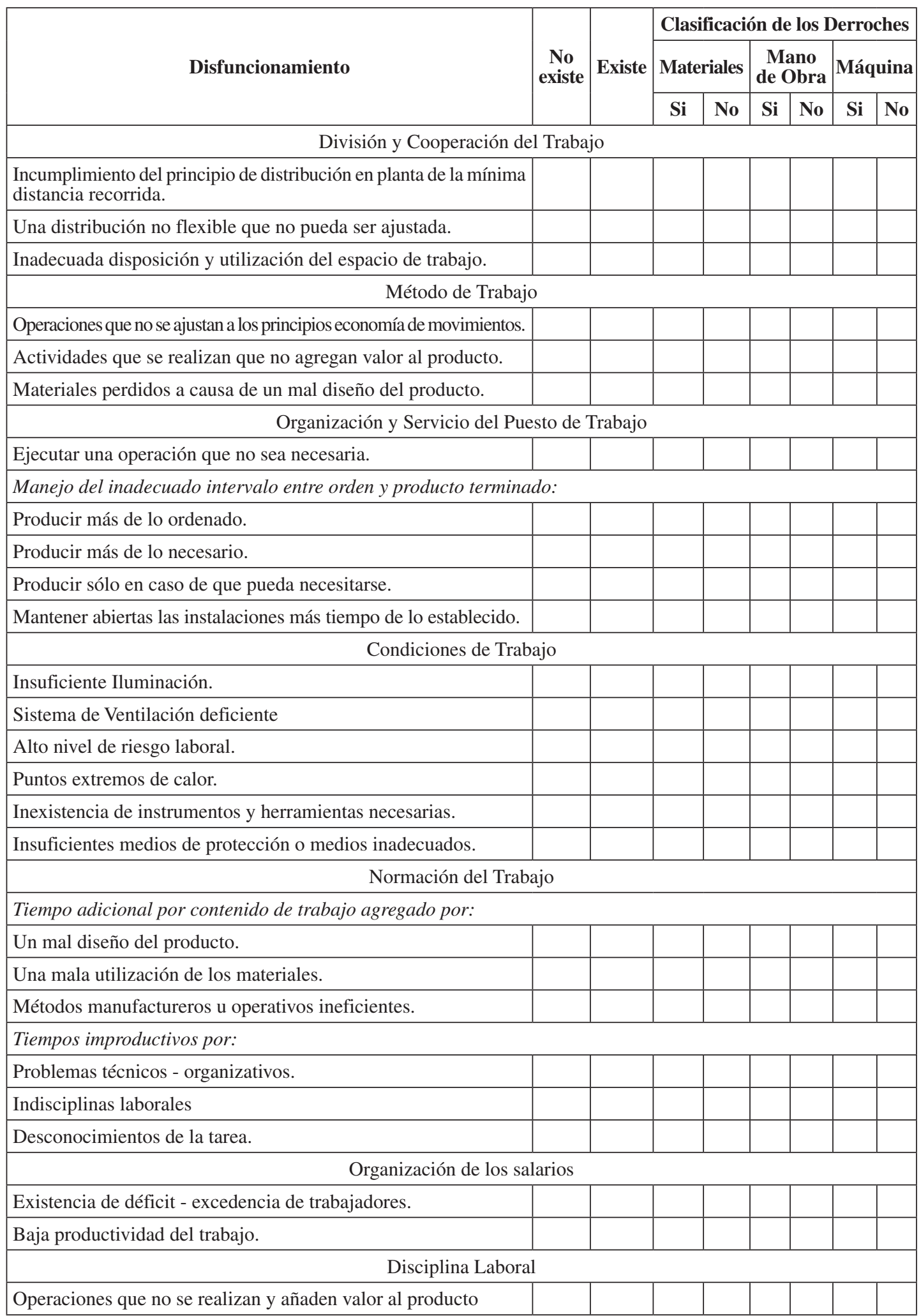


cumplimiento de los requisitos de la norma cubana 3002, 2007 [10].

Este procedimiento se realiza para cada una de las tres primeras etapas del procedimiento como se muestra en la Figura 1. Para lo cual se propone una ficha de proceso que agrupe todas estas actividades con un conjunto de indicadores para evaluar el cumplimiento y nivel efectividad de las mismas.

A partir de los resultados anteriores se propone realizar un informe con la propuesta de las soluciones

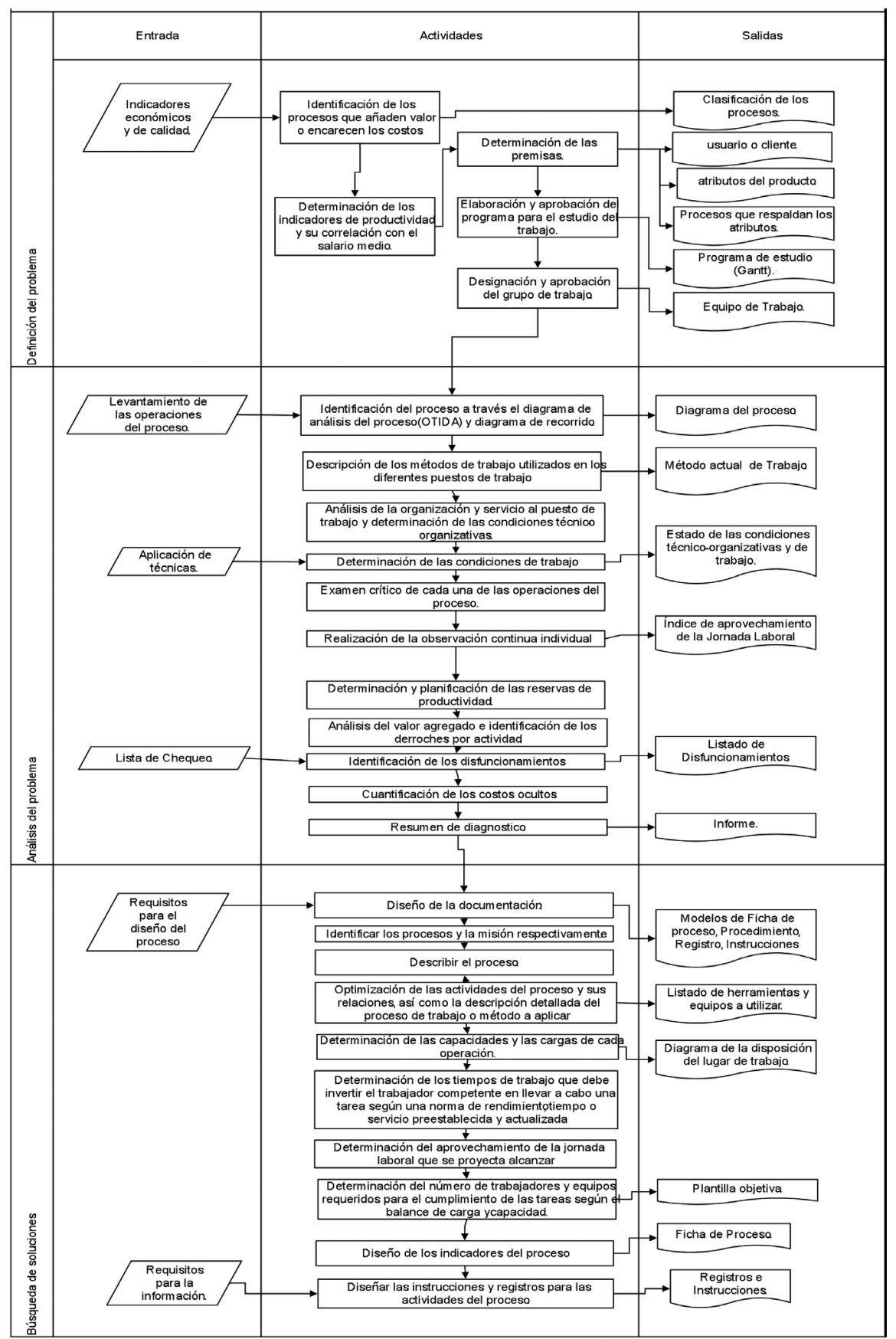

Figura 1. Procedimiento para el diseño del proceso de organización del trabajo. 
y exponerlas al colectivo de trabajadores para evaluar las alternativas de solución y así se complementa el requisito 4.3.8.

\section{Evaluación de alternativas de solución}

Se realiza la elaboración de informe de propuesta de soluciones y exposición de los resultados al colectivo de trabajadores para evaluar las alternativas de solución que contemplan el requisito 4.3.8.

\section{Selección de alternativas de solución}

Requisito 4.3.9: A partir de los resultados del balance de carga y capacidad y la optimización de plantilla de cargos, según los indicadores y los procedimientos establecidos en la legislación, con la aprobación de la dirección.

\section{Implementación de la solución}

Implementando el requisito 4.3.10, se aplica la plantilla de cargos aprobada y demostrar su correspondencia con el nivel de la actividad productiva o de servicios que desarrolla la organización.

\section{Evaluar efectividad y rediseñar}

Requisito 4.3.11. En este paso se determinan los indicadores de productividad y su correlación con el salario medio para evaluar si son positivos respecto a la ejecución de períodos anteriores, así como las acciones a seguir para garantizar la participación de los trabajadores en el análisis y diseño de las medidas y su control.

Los indicadores expuestos en las ecuaciones (1), (2), (3) se referencia de la investigación realizada por Marsán, A. Cuesta, C. García y C. Padilla [6]. Además, se diseñan los indicadores propuestos en las ecuaciones (4), (5), (6) y (7):

$$
\text { Productividad }=\frac{\text { Volumen de Producción }}{\text { Cantidad de Trabajo invertido }}
$$

$$
\begin{aligned}
\text { Índice de ausentismo }= & \frac{\text { Índice de días de ausencias }}{\left(\frac{\text { Total de plantilla }}{100}\right)} \\
& * \text { Total de días laborales }
\end{aligned}
$$

$$
\underset{\text { Índice de }}{\text { aprovechamientode la JL }}=\frac{\mathrm{TTR}+\mathrm{TIR}}{\mathrm{JL}} * 100
$$

Donde:

JL: Jornada Laboral.

TTR: Tiempo de Trabajo Reglamentado.

TIR: Tiempo de Interrupciones Reglamentadas.

$$
\begin{gathered}
\text { Índice de procesos } \\
\text { que añaden valor }
\end{gathered}=\frac{\begin{array}{c}
\text { Total de procesos que } \\
\text { añaden valor }
\end{array}}{\text { Total de procesos }}
$$

$$
\begin{array}{cc}
\begin{array}{c}
\text { Índice de estudios } \\
\text { de EOT realizados } \\
\text { en procesos que } \\
\text { añaden valor }
\end{array} & \begin{array}{c}
\text { realizados en procesos } \\
\text { que añaden valor }
\end{array} \\
\text { Total de procesos que } \\
\text { añaden valor }
\end{array}
$$

$\begin{gathered}\text { \% de implementación } \\ \text { de los resultados EOT }\end{gathered}=\frac{\begin{array}{c}\text { Cantidad de acciones } \\ \text { de los resultados EOT }\end{array}}{\begin{array}{c}\text { implementados } \\ \text { Cantidad de acciones } \\ \text { generadas a partir } \\ \text { de EOT realizados }\end{array}} * 100(7)$

Donde:

EOT: Estudio de Organización del Trabajo.

\section{RESULTADOS}

El procedimiento se aplica en una empresa del sector farmacéutico, donde se hace énfasis en la segunda etapa de análisis del problema para la identificación de los disfuncionamientos y la cuantificación de los costos ocultos existentes. A continuación, se muestran los principales resultados obtenidos:

En la identificación del problema se muestran como proceso que añaden valor: la producción de fármacos y para ello se realiza el mapa de procesos, donde los procesos claves son: la mercadotecnia, la producción de fármacos y las ventas. Los clientes externos son lo que hacen uso de estos fármacos 
y los atributos de estos procesos radican en la satisfacción del cliente, en la calidad de los fármacos y la efectividad de los mismos.

La empresa tiene una plantilla de 315 trabajadores, donde el $63 \%$ son técnico y el $23 \%$ operarios y el resto de servicio y administrativos. A partir del análisis de documentos, específicamente del informe económico de la entidad de los años 2013 y 2014 se examina el comportamiento de los indicadores de eficiencias del año 2014 con respecto al 2013, como se muestra en la Tabla 2. Estos indicadores revelan un resultado muy favorable, donde los ingresos han aumentado en un $9 \%$ y las ventas netas en un $8 \%$, así como la productividad del trabajo van en aumento. Aunque es importante destacar que se muestra un aumento del Total de Costos y Gastos en un 1.748 Miles de Unidades Monetarias (MUM).

A partir del análisis de los indicadores y de una entrevista realizada a varios directivos de la entidad, los cuáles plantean que existen problemas de indisciplinas laborales y lo cuál generan costos en la producción que no se cuantifican. Esta entrevista se diseñó a partir del objetivo de la investigación y posteriormente se procesó a través del Software MINITAB. A partir de los resultados obtenidos se decide realizar un estudio de organización del trabajo en el proceso de producción, para lo cual se reúne un equipo de trabajo con 7 expertos en el tema y el mismo se planifica mediante un diagrama de Gantt utilizando el Microsoft Project.

Durante el análisis crítico del proceso de organización del trabajo se detecta que en la empresa no están planificados los estudios de organización del trabajo, ni están aprobadas las herramientas y técnicas con que se realizaran en los mismos, así como que no cuenta con una descripción de los procesos.

La descripción escrita para los nuevos procesos o métodos resultantes de la aplicación de estudios del trabajo no contiene: las herramientas y equipos que se utilizarán, así como condiciones de trabajo, de seguridad y salud en el trabajo y ergonómicas a garantizar, diagrama de la disposición del lugar de trabajo, posible croquis de las herramientas, plantillas y otros dispositivos y solo en algunos casos están determinados los niveles de aprovechamiento de la jornada laboral.

La plantilla de cargos no está respaldada por un estudio de organización del trabajo, sino que se realiza mediante el análisis de la operatividad del proceso.

En el análisis del problema se parte del estudio del proceso, representando todas sus actividades como se muestra en la Figura 2, mediante un diagrama OTIDA.

El proceso comienza con la realización de la solicitud de la materia prima en dependencia del volumen de producción a elaborar. Estas materias primas pasan al área de preparación en donde se realiza la mezcla de las materias primas en los reactores según su tecnología. Al producto elaborado se le realizan los análisis correspondientes según sus características. Si corresponden con los requisitos de calidad se continúa el proceso productivo.

Una vez que el producto cumple con las especificaciones de calidad se pasa a tanques reservorios para su posterior envase y etiquetado. Cuando es dada la orden, pasa al área de envase y se realiza

Tabla 2. Comportamiento de los indicadores de eficiencia de la empresa.

\begin{tabular}{|l|c|c|}
\hline \multicolumn{1}{|c|}{ Indicador } & Año 2013 & Año 2014 \\
\hline Total, de Ingresos (MUSD). & $23.358,84$ & $25.422,10$ \\
\hline Ventas Netas (MUSD). & $24.158,00$ & 26.220 \\
\hline Eficiencia del producto terminado (\%). & 96 & 98 \\
\hline Total, de Costos y Gatos (MP). & $21.308,04$ & $23.056,60$ \\
\hline Costo por peso Total (MUSD). & 0,9122 & 0,9070 \\
\hline Valor Agregado Bruto (MUSD). & 13.336 & 15.637 \\
\hline Productividad Mensual S/VA (MUSD) & 50.326 & 57.915 \\
\hline Correlación Salario Medio - Productividad (MP). & 0,8835 & 0,8800 \\
\hline
\end{tabular}




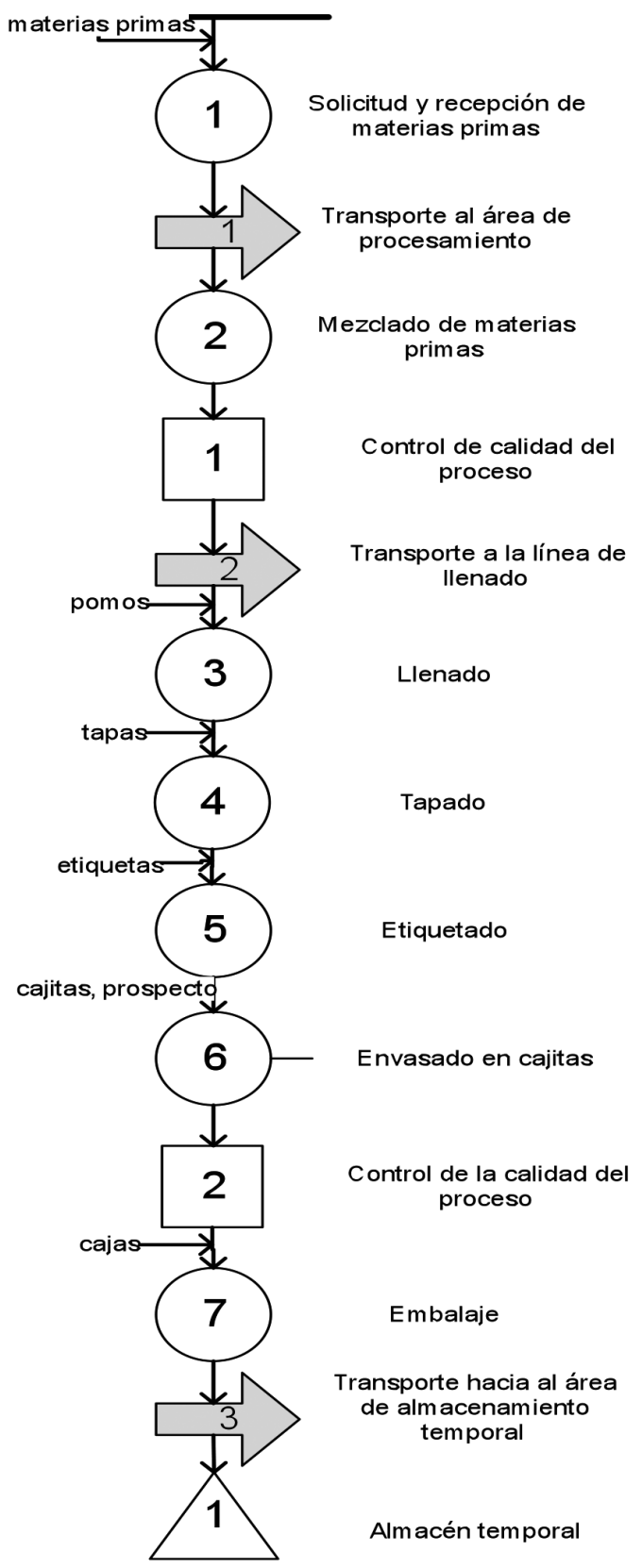

Figura 2. Diagrama de proceso. el mismo en frascos de polietileno de $500 \mathrm{ml}$, con un peso de $0,66 \mathrm{~kg}$ aproximadamente, el cual se identifica con su etiqueta correspondiente, todos estos materiales son importados. Posteriormente una muestra del producto terminado va al laboratorio para hacerle una serie de análisis de calidad para conocer si cumple con los parámetros necesarios. Luego se envasa en un estuche de cartón con el prospecto del producto, se embalan en cajas de cartón con capacidad de 16 estuches y se agrupan en paquetes de 60 cajas. Seguidamente pasa al almacén de productos terminados donde se almacena, según las normativas de almacenamiento hasta que exista disponibilidad en los almacenes centrales.

A partir de la identificación de las actividades del proceso se realiza un estudio de tiempo aplicando la herramienta muestreo del trabajo con el objetivo de conocer las causas que provocan las indisciplinas laborales de los técnicos, causa fundamental del problema planteado por los expertos.

El muestreo se aplicó a 25 técnicos del área de producción con un nivel de confianza del $95 \%$ y precisión $(\mathrm{S})= \pm 10 \%$. Se realizó durante 4 días con 4 recorridos diarios en instantes aleatorios dentro de la jornada laboral, para ello se parte de 100 observaciones iniciales, los resultados se muestran en la Tabla 3.

En el muestreo se obtiene un porcentaje de inactividad de un $43,7 \%$ considerado alto según el tipo de actividad que se desarrolla.

La sigma calculada es igual a $3,75 \%$, a partir de ello se calculan los límites del gráfico control diario que son: Límite Central $(\mathrm{LC})=43,86$, Límite Central Superior $($ LCS $)=55,11$ y Límite Central Inferior $(\mathrm{LCI})=32,61$. Todo esto permite detectar si el valor de Pd obtenido durante un día cualquiera se ha salido de la normalidad.

Tabla 3. Resultados del muestreo del trabajo en el proceso de producción.

\begin{tabular}{|l|r|r|r|c|}
\hline \multirow{2}{*}{ Datos } & \multicolumn{4}{c|}{ Días } \\
\cline { 2 - 5 } & $\mathbf{1}$ & $\mathbf{2}$ & $\mathbf{3}$ & $\mathbf{4}$ \\
\hline $\mathrm{P}$ (Observaciones P computadas el día d) & 50 & 92 & 80 & 85 \\
\hline $\mathrm{N}($ Observaciones totales realizadas el día d). & 100 & 200 & 200 & 200 \\
\hline $\mathrm{P}_{\mathrm{d}}(\%)$ (Porcentaje de inactividad hasta el día d) & 50 & 46 & 40 & 42,5 \\
\hline $\mathrm{P}_{\mathrm{ad}}(\%)$ (Porcentaje de inactividad acumulado hasta el día d) & 50 & 47,3 & 44,4 & 43,86 \\
\hline
\end{tabular}


Este grafico sirve para comparar Porcentaje de inactividad acumulado final $\left(\mathrm{P}_{\mathrm{f}}\right)$ obtenida con los distintos valores de $\left(\mathrm{P}_{\mathrm{ad}}\right)$ obtenido cada día, para comprobar si son elementos de la distribución normal, correspondiente a la distribución muestral de la población que estamos analizando.

La precisión final (Sf) es de 8,38\%. Por lo tanto, comparando precisión inicial fijada $(S)= \pm 10 \%$. tanto, comparando Sf con la S inicial se obtiene $S_{f}=8,38 \%$ $<S=10 \%$, lo cual significa que se acepta el muestreo.

La inactividad final de los trabajadores es de un $43,86 \%$ y calculando el aprovechamiento de la jornada laboral según la descripción de las actividades realizada y los tiempos clasificados durante el muestreo es de un $70 \%$, lo cual es bajo respecto al tipo de actividad, es por esto que se hace necesario realizar un análisis de las principales causas del no aprovechamiento de la jornada laboral, para ello se realiza un Diagrama de Pareto partiendo de las frecuencia de incidencia de estas causas registradas durante el muestreo, las cuales se procesan utilizando el Software MINITAB. Este resultado se muestra en la Figura 3.

En el gráfico de Pareto se puede apreciar que, aunque no se cumple la relación 80-20, la principal causa es la existencia de indisciplinas laborales y si estas son eliminadas se resuelve más del $60 \%$ del problema.

Las indisciplinas laborales están asociadas a disfuncionamientos que pueden existir en los

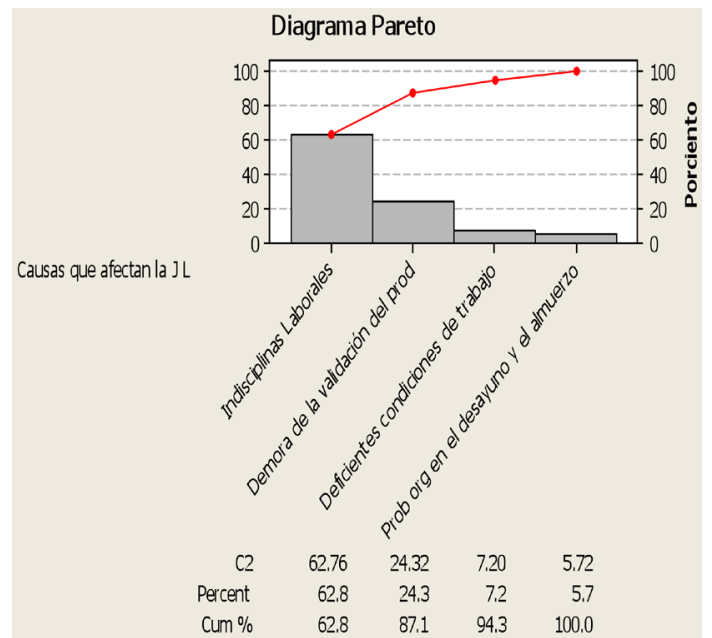

Figura 3. Diagrama de Pareto. procesos y para detectar los mismos se aplica la lista de chequeo presentada anteriormente en la Tabla 1.

La lista de chequeo se procesa mediante un método de expertos y para ello se selecciona un grupo de personas que laboran en el proceso. A los cuáles mediante una encuesta diseñada para evaluar si eran expertos y su índice de experticia, se logra seleccionar 8 expertos, de ellos 6 fueron categorizados como expertos altos por tener un coeficiente de competencia superior a $0,8(0,8 \leq \mathrm{K}$ $\leq 1)$, y 2 clasificados como medio $(0,5 \leq K<0,8)$. El coeficiente de competencia promedio de los expertos seleccionados resulta de 0,86 .

Posteriormente se determinó la concordancia entre los expertos sobre cuáles eran los disfuncionamientos existentes, determinándose una concordancia superior al $60 \%$, por se obtienen 7 disfuncionamientos.

En la tercera ronda se determinó el nivel de incidencia del disfuncionamiento en la existencia de derroches o costos ocultos en el proceso, ponderándolos de 1 a 12 donde 1 es el que más incide y el 12 es el menos incide.

A partir de estas votaciones se procesa la información con el objetivo de buscar el consenso entre los expertos, para ello se utiliza la Prueba de Concordancia de Kendall (W) [7]. En este caso el número de expertos es 8 y la cantidad de disfuncionamiento es igual a 7 , por lo que el consenso de los expertos, que es suma de los cuadrados de las desviaciones observadas de la media de $\mathrm{Rj}$, en la ecuación (8) se calcula la sumatoria de los índices de importancia establecidos por los expertos y combinando las ecuaciones anteriores se obtiene la ecuación (9). En la ecuación (10) se realiza la comparación con la S establecida en la región crítica:

$$
\bar{R}=\frac{\sum R j}{N}
$$

donde,

$$
S=\sum_{i=1}^{n}\left(\frac{R_{j}-\sum R_{j}}{N}\right)^{2}=63504
$$


Hipótesis

H0: el juicio de los expertos no es consistente. H1: el juicio de los expertos es consistente.

Región crítica:

$$
\begin{aligned}
& S>S_{7 ; 8}^{*} \\
& 63504>453.1
\end{aligned}
$$

Se realizó la búsqueda en la tabla de valores críticos de concordancia de Kendall. Con la prueba estadística calculada se obtiene $\mathrm{H} 1$ por tanto se puede concluir que el juicio de los expertos es consistente.

En la Figura 4 se muestra el porcentaje de experto que votaron por la existencia de estos disfuncionamientos.

Los principales disfuncionamientos como se muestra en la Figura 4 son: la inexistencia de instrumentos y herramientas necesarias con un $31 \%$ de expertos de acuerdo con su existencia y los tiempos improductivos por indisciplina laboral con un $26 \%$.

En el diagnóstico realizado se determinó que existe un aprovechamiento de la Jornada laboral de un 70\% lo cual es bajo por el tipo de trabajo que se realiza.

El disfuncionamiento asociado a las indisciplinas laborales fue cuantificado por el método de costeo

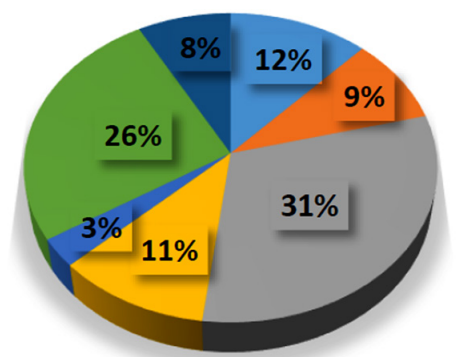

Una distribución no flexible que no pueda ser ajustada.

Actividades que se realizan que no agregan valor al producto.

- Inexistencia de instrumentos y herramientas necesarias.

- Métodos manufactureros u operativos ineficientes.

口 Problemas técnicos - organizativos.

- Tiempos improductivos por indisciplinas laborales

Existencia de déficit - excedencia de trabajadores.

Figura 4. Porcentaje de expertos que votaron por la importancia la existencia de los disfuncionamientos.
ABC como se muestra en la Tabla 4, a través de los ítems que componían el mismo, que son: falta de motivación por parte de los trabajadores, falta de control de jefes inmediatos mientras los trabajadores conversan en la Jornada Laboral (JL) y no trabajan, así como el régimen de trabajo y descanso extendido fuera de los 30 minutos establecidos como tiempo de descanso y necesidades personales.

Con el objetivo de evaluar la efectividad de estas soluciones se realiza el análisis de los indicadores propuesto determinándose en las ecuaciones (1), (2), (3), (4), (5), (6) y (7). Para ello se realizó una comparación entre los años 2014 y 2015 de acuerdo al momento de aplicación de este procedimiento. Los resultados se muestran en la Tabla 5.

En la Tabla 5 muestra como los indicadores aumentan su comportamiento, a partir de que se disminuyen las indisciplinas laborales, y esto genera un aumento del porcentaje del aprovechamiento de la Jornada Laboral.

Tabla 5. Cuantificación de los costos ocultos.

\begin{tabular}{|l|c|c|}
\hline \multirow{2}{*}{ Indicador } & \multicolumn{2}{c|}{ Años } \\
\cline { 2 - 3 } & $\mathbf{2 0 1 4}$ & $\mathbf{2 0 1 5}$ \\
\hline $\begin{array}{l}\text { Implementación de los resultados } \\
\text { de estudios de OT. }\end{array}$ & $20 \%$ & $50 \%$ \\
\hline $\begin{array}{l}\text { Cumplimiento del cronograma de } \\
\text { los estudios de OT. }\end{array}$ & $30 \%$ & $70 \%$ \\
\hline Índice de ausentismo. & $6 \%$ & $5 \%$ \\
\hline Índice de AJL. & $60 \%$ & $85 \%$ \\
\hline $\begin{array}{l}\text { Estudios de OT realizados en los } \\
\text { procesos que añaden valor. }\end{array}$ & $57 \%$ & $98 \%$ \\
\hline $\begin{array}{l}\text { Índice de procesos que añaden } \\
\text { valor a la organización. }\end{array}$ & $20 \%$ & $15 \%$ \\
\hline Productividad Mensual (MUSD). & 6032,2 & $6.064,2$ \\
\hline $\begin{array}{l}\text { Correlación Salario Medio - } \\
\text { Productividad (MP). }\end{array}$ & 0,880 & 0,920 \\
\hline
\end{tabular}

En las soluciones a los problemas encontrados se realizó un cronometraje con tiempo por método estadístico, determinando los tiempos para cada operación del proceso.

En la operación de etiquetado se realizó una mejora en el método de trabajo con el fin de optimizar las actividades, eliminando dos actividades innecesarias, 
esto contribuye a reducir el tiempo de operación de 20 minutos a 15 minutos lo que conlleva un aumento en la productividad del trabajo respecto al método anterior de un $5 \%$.

En el nuevo diseño de la organización del trabajo se aplicó el procedimiento propuesto en la Figura 1, diseñado los registros y actividades correspondientes al proceso.

\section{CONCLUSIONES}

En la investigación se ajustó el procedimiento de Fleitas y García [26] para el estudio de organización del trabajo con un enfoque económico, al añadirle la herramienta para la identificación de disfuncionamientos y como se cuantifican los costos ocultos, por lo que se distingue por integrar aspectos sociales a los económicos.

Con la aplicación del procedimiento se logra cuantificar que los costos ocultos asociados a los tiempos improductivos por indisciplinas laborales ascienden a 1857 pesos cubanos por mes por trabajador en el proceso de producción de la empresa del sector farmacéutico.

Se diseña y se aplica el proceso de organización del trabajo según los requisitos de la Norma Cubana: 3002, 2007, así como indicadores para controlar el proceso, lo que permite realizar un estudio a profundidad que no sólo permite el ahorro de dinero a la organización, sino que a través de la mejora continua se pueden lograr un máximo de rendimiento que se traduce en mejores indicadores de los procesos.

\section{REFERENCIAS}

[1] H. Savall y V. Zardet. "Reciclar los costos ocultos durables: la gestión socioe-conómica. Método y resultados". Administración y Organizaciones. Vol. 16, $\mathrm{N}^{\circ}$ 8, pp. 17-43. Junio 2006. ISSN: 1665-014X. URL: http:// bidi.xoc.uam.mx. Fecha de Consulta: 20 de enero de 2014.

[2] G. Salvendy. "Handbook of Industrial Engineering: Technology and Operations Management". John Wiley \& Sons Incorporated. Edition 3, pp. 249-268. United States of America. ISBN: 9780470241820. 2007.
[3] G. Kanawaty. "Introducción al estudio del trabajo". Ed. Oficina Internacional del Trabajo. 4ta edición. Ginebra, Suiza, pp. 22-34. 1996. ISBN: 92-2-307108-9.

[4] B.W. Niebel y A. Freivalds. "Ingeniería Industrial. Métodos, estándares y diseños del trabajo". Ed. McGraw-Hill. 12ma edición, pp. 19-36. México. 2004. ISBN: 978-97010-6962-2. 2004.

[5] W. Hondson "Maynard. Manual del Ingeniero". Ed. McGraw-Hill, 4ta edición. México. Vol. 1, pp. 43-55. 2001 ISBN 978-970-10-1195-9.

[6] J.R. Marsán, A. Cuesta, C. García y C. Padilla. "Organización del Trabajo. Ingeniería de Métodos". Ed. Félix Varela, 2da edición. La Habana, Cuba, pp. 3-35. 2011. ISBN: 978-959-07-1420-7.

[7] A. Morales "Capital Humano, hacia un sistema de gestión en la empresa cubana". Ed. Política. 1era edición. La Habana, Cuba. pp. 12-34. 2009. ISBN: 978-959-01-0855-6. 2009.

[8] "Oficina Nacional de Normalización: Norma Cubana: Sistema de gestión integrada de capital humano - 3000 Vocabulario". Ed. Oficina Nacional de Normalización (NC). La Habana, 2007. URL: www.nc.cubaindustria.cu

[9] "Oficina Nacional de Normalización: Norma Cubana: Sistema de gestión integrada de capital humano-Requisitos -3001", Ed. Oficina Nacional de Normalización (NC). La Habana, Cuba. 2007.

[10] "Oficina Nacional de Normalización: Norma Cubana: Sistema de gestión integrada de capital humano-Implementación-3002", Ed. Oficina Nacional de Normalización (NC). La Habana, Cuba. 2007.

[11] I. Hernández, S. Fleitas y D. Salazar. "Particularidades de la gestión de los recursos humanos en empresas cubanas". Revista Avanzada Científica. Vol. $14 \mathrm{~N}^{\circ}$ 1, pp. 35-46. Marzo-Abril, 2011. ISSN: 1029-3450. URL: https://dialnet.unirioja.es/descarga/ articulo/3646564.pdf. Fecha de Consulta: 25 de enero de 2014.

[12] A. Cuestas Santos. "Modelo integrado de gestión humana y del conocimiento: una tecnología de aplicación" Revista Venezolana de Gerencia (RVG). Año 17 No 57, pp. 86 - 98. Enero-Marzo, 2012 ISSN 1315-9984. 
URL: http://www.redalyc.org/articulo. oa $?$ id=29021992005. Fecha de Consulta: 25 de febrero de 2014.

[13] A.M. Negrón González, SM Fleitas, F Oro y A. M. González. "Proceso de selección del personal docente en la Facultad de Ingeniería Industrial del Instituto Superior Politécnico José Antonio Echeverría de La Habana, Cuba". Opción: Revista de Ciencias Humanas y Sociales, Año $30 \mathrm{~N}^{\circ} 73$, pp. 101118. Enero - Abril, 2014, ISSN 1012-1587. URL: http://www.redalyc.org/articulo. oa? $i d=31035398002$. Fecha de Consulta: 1 de abril de 2014.

[14] E. Marianao, E. Torres y C. Chasco. "Satisfacción: determinante de la familiaridad del destino turístico". Revista de Administração de Empresas (RAE). Vol. $54 \mathrm{~N}^{\circ}$ 4, pp. 393-404. Julio-Agosto, 2014. ISSN 0034-7590. URL: www.redalyc. org/pdf/1551/155131348008.pdf. Fecha de Consulta: 1 de abril de 2014.

[15] J. Cardozo, B. Hernandis y N. Ramírez. "Caracterización de los sistemas de productos en el marco de la personalización, la variabilidad y la diferenciación. Un estudio con expertos". Ingeniare. Revista chilena de ingeniería. Vol. 22, $\mathrm{N}^{\circ} 2$, pp. 393-404. Abril - Junio, 2014. ISSN 0718-3305. URL: http://dx.doi.org/10.4067/S071833052014000200013. Fecha de Consulta: 1 de noviembre de 2014.

[16] A. Erbes, S. Roitter y M. Delfini. "Organización del trabajo e innovación: Un estudio comparativo entre ramas productivas argentinas". Revista Economía: teoría y práctica. $\mathrm{N}^{\circ} 34$, pp.101-132, Enero - Junio, 2011 ISSN 0188-8250. URL: http://www. scielo.org.mx/pdf/etp/n34/n34a5.pdf

[17] M.C. Sánchez Sellero, P. Sánchez Sellero, M. M. Cruz González, F.J Sánchez Sellero. "Características organizacionales de la satisfacción laboral en España". Revista de Administração de Empresas (RAE). Vol. 54 $\mathrm{N}^{\circ}$ 5, pp.537-547. Septiembre-Octubre, 2014. ISSN 0034-7590. URL: www.scielo.br/pdf/ rae/v54n5/0034-7590-rae-54-05-0537.pdf

[18] E.J. Hernández, Z.M. Camargo y P. M.T. Martínez. "Impact of $5 \mathrm{~S}$ on productivity, quality, organizational climate and industrial safety in Caucho Metal Ltda”. Ingeniare.
Revista chilena de ingeniería, Vol. $23 \mathrm{~N}^{\circ} 1$, pp. 107-117, Enero - Marzo, 2015. URL: http:// www.ingeniare.cl/index.php?option $=\mathrm{com}_{-}$ ingeniare $\&$ view $=\mathrm{d} \& \mathrm{doc}=82 / \operatorname{art} 13$. pdf \&aid=410\&vid=82\&lang=es.Fecha de Consulta: 5 de noviembre de 2014.

[19] A. Ollo-López, A. Bayo-Moriones y M. Lazarra-Kintana. "Perfil de los empleados involucrados en las nuevas prácticas de Organización del Trabajo". Cuadernos de Economía y Dirección de la Empresa. $\mathrm{N}^{\circ} 39$, pp. 095-122, Junio, 2009 ISSN: 1138-5758. URL: www.elsevier.es/ es-revista-cuadernos-economia-direccionempresa-cede-324-pdf-S1138. Fecha de Consulta: 2 de noviembre de 2014.

[20] A.C. Fleury, L.Snelwar, R. Marx, M, Silva y M. Zibovicius. "Prospecção de Mudanças Ocupacionais Decorrentes de Novas Formas de Organização do Trabalho: têxtil, petroquímico, telecomunicações e máquinas e equipamentos". Edit. SENA, Brasilia, Brasil. Vol. 2, pp. 13-28. 2006. ISBN 85-7519-186-1.

[21] H. Hirata, C. Ferreira, R. Marx y M. Salerno. "Gestão da qualidade: tecnologia e participação".Edit CODEPLAN, Brasilia, Brasil. Vol. 1, pp. 157-178. 1992.

[22] J.Olivella, L. Cuatrecasas y N. Gavilan, $\mathrm{N}$. "Work organisation practices for lean production". Journal of Manufacturing Technology Management. Vol. 19, $\mathrm{N}^{\circ} 7$, pp. 798- 811. 2004. ISSN 1741-038X. URL: http://www.emeraldinsight.com/doi/ abs/10.1108/17410380810898750.DOI: http:// dx.doi.org/10.1108/17410380810898750. Fecha de Consulta: 4 de noviembre de 2014.

[23] G.S. Liu, R. Shah y R.G Schroeder. "Linking work design to mass customization: A sociotechnical systems perspective". Decision Sciences. Vol. $37 \mathrm{~N}^{\circ}$ 4, pp. 519545, Noviembre, 2016 ISSN 0011-7315. URL: http://onlinelibrary.wiley.com/ doi/10.1111/j.1540-5414.2006.00137.x/ full. DOI:10.1111/j.1540-414.2006.00137.x Fecha de Consulta: 1 de noviembre de 2014

[24] J. Basnuevo. "Procedimiento para la realización de estudios de organización del trabajo en empresas productivas". Tesis para optar por el título de máster. Universidad Tecnólogica de La Habana, Cujae, La Habana, Cuba. 2008. 
[25] J. Bernal y L. Ramos. "Procedimiento para el estudio de la organización del trabajo en empresas cubanas". Revista Avanzada Científica. Vol. $5 \mathrm{~N}^{\circ} 1$, pp. 63-69. Enero - Abril, 2012. ISSN: 1029-3450. URL: https://dialnet. unirioja.es/descarga/articulo/4059838.pdf. Fecha de Consulta: 12 de noviembre de 2014.

[26] M.S. Fleitas y V. García. "Procedimiento para el estudio de la organización del trabajo". Revista Nueva Empresa, Vol. 9 N $^{\circ}$ 4, pp. 122135. Enero-Abril, 2013. ISSN: 2220-4148.

[27] S. Henri y Z.Veronique. "Ingeniería Estratégica, Un Enfoque Socioeconómico". Edit. Universidad Autónoma Metropolitan. 1era edición. México D.F, México. pp. 2-40. 2009. ISBN 978-607-477-063-6.
[28] A.M. Negrón González, M.S. Fleitas, J.C. Negrón e Y. Trujillo. "Identificación de los costos ocultos asociados al proceso de selección del personal". 17 Convención de Ingeniería y Arquitectura 2014. La Habana, Cuba. ISBN: 978-959-261-467-3. Noviembre, 2014.

[29] I.J. Rodríguez, A. González, P. Noy y S.Pérez. "Metodología de Diseño Organizacional integrando enfoque a procesos y competencias". Revista Ingeniería Industrial, Vol. XXXIII. No 2 , pp. 188-199. MayoAgosto, 2012. ISSN: 1815-5936. URL: http:// scielo.sld.cu/scielo.php?script=sci_arttext\& pid=S1815-59362012000200010. Fecha de Consulta: 20 de noviembre de 2014. 